\author{
Manpal Singh Bhogal \\ Department of Psychology, Institute of Human Sciences, University of \\ Wolverhampton, United Kingdom
}

\title{
Altruism Advertises Cooperativeness
}

\section{Synonyms}

Prosocial Behavior

\section{Definition}

Altruism refers to benefit provisioning behaviour, and cooperation is defined as exchange between two or more individuals which is mutually beneficial.

\section{Introduction}

Altruism is defined as a behaviour that is beneficial to a receiver, but costly to the altruist (Trivers, 1971). Altruism is a phenomenon which causes evolutionary theorists trouble when tying it into the overall play of evolution. Why be altruistic to someone you do not know?

The reason why altruism is a puzzling phenomenon is because it is costly in nature. Being altruistic has also been argued to be costly to an individual's fitness, beneficial to the receiver's fitness but at a cost to the giver. Because of the fitness deficit between altruist and recipient, Sanchez and Cuesta (2005) argue that altruism should therefore cease to exist over time if it provides us with no direct evolutionary 
advantage(s). However, we observe altruism in everyday life; giving up one's seat for an elderly person on public transport, helping someone by carrying their shopping, or stopping to help someone in an accident.

Evolutionary theorists have spent decades exploring the psychological mechanisms that drive altruism towards those we are not related to (see Kurzban, Burton-Chellew \& West, 2015 for a recent review). According to the principles of natural selection, altruism must have evolved in order to convey benefits to others, and to increase one's fitness, otherwise, due to its costly nature, it should not have evolved.

There are several explanations to explain the signalling purpose of altruistic behavior. Explanations derive from individual selection where the altruist expects reward from his or her action (this suggests that altruism is selfish as opposed to selfless - see Ruse, 2012). Further explanations derive from indirect and direct reciprocity (Roberts, 1998), where altruistic behaviour evolves through engaging cooperatively with others. Reputation building has also been proposed (Nowak \& Sigmund, 1998), where altruism increases one's reputation through continued cooperation with others, thus suggesting a good reputation can be sought from displays of altruism, which signal cooperative intent towards others. Multi-level selection has been used to explain the evolution of altruistic behaviour, arguing that natural selection, when operated on a group level, increases the survival of a group. For example, if a group contains more altruists compared to other groups, it increases the likelihood that the group which contains altruistic people will outperform and have increased longevity compared to the group of non-altruists. In relation to cooperativeness, although altruism (at face value) is a costly endeavour, multi-level selection diminishes the personal cost of altruistic behaviour, as altruism 
may advertise cooperativeness through multi-level selection by increasing the survival of a group (Wilson, 2015).

Altruism is crucial, on a societal and individual basis, as we need to be both on the receiving end of altruism and be engaging in displays of altruistic to survive, particularly through being chosen as partners in cooperative ventures (Ruse, 2012). Throughout our evolutionary history, cooperation has been crucial in increasing survival. Even today, cooperation is just as important to governments maintaining relationships with one another, with kin or non-kin alike. The decisions we make in everyday life, whether they involve family, friends, acquaintances, enemies or strangers with whom you are walking along the street, are influenced by conflict and cooperation. Day to day decisions such as deciding whether to confront a colleague about taking advantage of your good nature or deciding whether to deal with the free rider in a group task, are both influenced by cooperation or conflict.

For altruism to evolve, the benefits associated with these traits should provide benefits through reciprocating altruistic behaviour, leading to cooperation (Kurzban et al., 2015). If the benefits of cooperation are greater than the cost of cooperation, benefits can be accrued by continuously cooperating with those who cooperate with us.

Cooperative tendencies refer to a variety of behaviours and traits. For example, cooperation can encompass altruism, furthering the notion that altruistic behaviour signals, or advertises cooperative intent (Baumard, André \& Sperber, 2013). Reciprocal altruism (Trivers, 1971) suggests that people are altruistic towards others when there is a mutual benefit in being so. This theory suggests that altruism is rewarded through reciprocation, suggesting the benefits of altruism somewhat 
match, or exceed the costs of engaging in the altruistic act (Trivers, 1971), implying the interaction is repeated, and the recipient will reciprocate in the future, thus leading to cooperative behaviour. One of the conditions set by reciprocal altruism is that the altruistic cost should provide a larger benefit to the receiver than the altruist. Manktelow (2012, p. 80) states "If you take a benefit, then you pay a cost". Evidence suggests that humans are not simply altruistic in order to gain immediate benefits, but altruistic behaviour may lead to immediate and/or future gains through cooperative ventures (Stevens \& Hausser, 2004).

Being cooperative may not always benefit an individual in the short-term and may in fact be costly in the short-term, but actors may 'recoup their losses in the future' (Stevens \& Hauser, 2004; p. 60). Reciprocal altruism has been extensively studied in a range of economic contexts, and due to the societal importance of cooperation, humans have developed cognitive functions to ensure cooperation is embedded into our society, which may also be a reason why cheater detection and free riders are (generally) punished in society. After all, in order to monitor social exchange fairly, and prevent others from reaping benefits without paying a cost, we must detect, and punish cheaters (Manktelow, 2012).

\section{Conclusion}

In summary, altruistic behaviour can often signal cooperative intent when communicating with others. This can be through direct observation, through reputation building, indirect, and direct reciprocity.

Cross-References: Altruism, Cooperation, Prosocial Behavior

\section{References}


Baumard, N., André, J., \& Sperber, D. (2013). A mutualistic approach to morality: The evolution of fairness by partner choice. Behavioral and Brain Sciences, 36, $59-122$

Kurzban, R., Burton-Chellew, \& West, S, A. (2015). The Evolution of Altruism in humans. Annual Review of Psychology, 66, 575-599.

Manktelow, K. (2012). Thinking and Reasoning: An introduction to the psychology of reason, judgment and decision making. Psychology Press. London and New York.

Nowak, M. A., \& Sigmund, K. (1998). Evolution of indirect reciprocity by image scoring. Nature, 393, 573-577.

Roberts, G. (1998). Competitive altruism: From reciprocity to the handicap principle. Proceedings of the Royal Society of London, 265, 427-431.

Ruse, M. (2012). The Philosophy of Human Evolution. Cambridge University Press.

Sanchez, A., \& Cuesta, J. A. (2005). Altruism may arise from individual selection. Journal of Theoretical Biology, 235, 233-240.

Stevens, J. R., \& Hauser, M. D. (2004). Why be nice? Psychological constraints on the evolution of cooperation. Trends in Cognitive Sciences, 8(2), 60-65.

Trivers, R. L. (1971). The evolution of reciprocal altruism. Quarterly Review of Biology, 46, 35-57.

Wilson, D. S. (2015). Does altruism exist? Culture, Genes, and the Welfare of others. Yale University Press. 